\title{
Impact of Sowing Dates on Drymatter Production, Partitioning and Yield in Lentil (Lens culinaris Medikus)
}

\author{
Ananya Baidya ${ }^{1 *}$, Anjan Kumar Pal ${ }^{1}$, Mohammed Anwar Ali ${ }^{2}$ and Rajib Nath ${ }^{3}$ \\ ${ }^{1}$ Department of Plant Physiology, ${ }^{3}$ Department of Agronomy, F/Ag, Bidhan Chandra Krishi \\ Viswavidyalaya, Nadia, West Bengal - 741252, India \\ ${ }^{2}$ Department of Crop Physiology, Acharya N.G. Ranga Agricultural University, Agricultural \\ College Bapatla, Andhra Pradesh-522101, India
}

*Corresponding author

A B S T R A C T

\begin{tabular}{|c|c|}
\hline Keywords & $\begin{array}{l}\text { Twenty lentil genotypes were taken to study the dry matter production, partitioning, and } \\
\text { vield, under three different dates of sowing }(15 \text { November, } 6 \text { December, and } 27\end{array}$ \\
\hline $\begin{array}{l}\text { Lentil } \\
\text { partiti } \\
\text { Late s }\end{array}$ & $\begin{array}{l}\text { December), data were recorded at various growth stages. A decrease in root dry matter in } \\
\text { late sowings at pre-flowering and post-flowering was observed. Crop grown on the second } \\
\text { sowing (late sown, } 6 \text { December) registered higher means for total dry weight and yield at }\end{array}$ \\
\hline Arti & \\
\hline $\begin{array}{l}\text { Accepted: } \\
\text { 26 March } 2018 \\
\text { Available Online: } \\
10 \text { April } 2018\end{array}$ & $\begin{array}{l}\text { reveals that reduced soil moisture and high air temperature, during reproductive stage led } \\
\text { to drastic reduction in the seed yield at third sowing (late sown, } 27 \text { December). The } \\
\text { promising genotypes under third sowing (late sown, } 27 \text { December) are PRECOZ and L-13- } \\
123 \text { as they maintained to yield higher as compared to other genotypes. }\end{array}$ \\
\hline
\end{tabular}

\section{Introduction}

Lentil is one of the most important winter grain legume crop in South Asia, West Asia, the Middle East, and South America. This crop ranks second in acreage and production, third in protein content in pulses grown in South Asia (SAIC, 2010). The major constraints responsible for low yield of lentil are short growth duration particularly slow rate of drymatter accumulation prior to flowering and unfavorable canopy structure (Mondal et al., 2013). In West Bengal, the transplanting of kharif paddy is often delayed by 3-4 weeks beyond the normal practice due to delayed and skewed rainfall pattern, as well as harvesting of kharif paddy is also delayed. Under these circumstances, farmers have no other alternative but to delay sowing of lentil by as long as one month or so in order to incorporate this pulse in the cropping sequence following paddy. Delayed sowing subjected to environmental factors such as very high temperature and drought stress which affects negatively on growth and yield of the crop. The crop flowers and matures 
under high temperature and terminal drought conditions.

\section{Materials and Methods}

The field experiment was conducted in District Seed Farm, AB Block, Kalyani, Nadia, West Bengal, India. The latitude is $22^{\circ} 58^{\prime} \mathrm{N}$ and longitude $88^{\circ} 32^{\prime} \mathrm{E}$ with an altitude of $9.75 \mathrm{~m}$ above mean sea level. The soil texture of the experimental plot was sandy loam with $\mathrm{pH}$ 6.9-7.0. Twenty genotypes of lentil were sown on three different dates viz., $15^{\text {th }}$ November, $6^{\text {th }}$ December and $27^{\text {th }}$ December 2014.

Each lentil genotype was sown in three replications in each case. The row length was $1.5 \mathrm{~m}$ and a spacing of $25 \mathrm{~cm}$ was followed in between rows while plant to plant spacing was $10 \mathrm{~cm}$. Fertilizers were applied at the rate of 20, 40 and $40 \mathrm{~kg} / \mathrm{ha} \mathrm{N}: \mathrm{P}: \mathrm{K}$. Appropriate plant protection measures along with other standard cultural practices were followed to raise a healthy crop. The crop was raised without application of any irrigation.

\section{Drymatter partitioning}

Data were recorded on drymatter content of whole plant and its partitioning to different plant parts. Plant sampling was done at 35 DAS (pre-flowering) followed by 50 DAS (flowering) and 60 DAS (post flowering). The whole plant was divided into several parts like root, shoot, leaf, reproductive plant parts (flower, bud, pod) and dry weight was recorded after drying the plant samples in the oven at $80^{\circ} \mathrm{C}$ till constant weight. Data were recorded on seed yield/plant at harvest.

\section{Data analysis}

The mean values for all the parameters were analyzed following split plot design with three replications by INDOSTAT version 7.1 software. In the experimental design, the dates of sowing were in the main plot and the lentil genotypes were taken in the sub plot.

\section{Results and Discussion}

Analysis of variance indicated the significant variations among sowing dates and genotypes for drymatter production and its partitioning to different plant parts at 35, 50 and 65 days after sowing (DAS). The interactions between sowing dates and genotypes also revealed significant differences in all the cases.

The mean values for the growth parameters showed that the dry weight of root at 35 and 65 DAS decreased under late sowing dates in comparison with that recorded under normal sowing date (Table 1). However, the root dry weight at 50 DAS registered the highest mean under $2^{\text {nd }}$ sowing date.

In case of drymatter content of shoot at 50 DAS, the crop sown on $6^{\text {th }}$ December also registered the higher mean values as compared to two other sowing dates. The higher mean of leaf dry weight at 35 and 65 DAS were recorded under $1^{\text {st }}$ sowing date as compared to late sowing dates in the present experiment, while the $2^{\text {nd }}$ sowing date was found to have superior performance in respect of shoot drymatter content at 50 DAS.

It was significant to note that the crop sown on $6^{\text {th }}$ December also registered higher means for total dry weight as well as drymatter content in reproductive parts (viz., flower, flower bud, and immature pods) at 50 and 65 DAS growth stages.

Perusal of genotypic means presented in Table 2 for drymatter production and its partitioning to different plant parts indicated that the drymatter of root and shoot registered steady increase with the progress of growth stages from 35 DAS to 65 DAS. 
Table.1 Main effects of sowing dates on total drymatter content and its partitioning and seed yield in lentil

\begin{tabular}{|c|c|c|c|c|c|c|c|c|c|c|c|c|c|c|c|c|}
\hline \multirow[t]{2}{*}{$\begin{array}{l}\text { Date of } \\
\text { sowing }\end{array}$} & \multicolumn{3}{|c|}{ Root DW (g) } & \multicolumn{3}{|c|}{ Shoot DW (g) } & \multicolumn{3}{|c|}{ Leaf DW (g) } & \multicolumn{3}{|c|}{$\begin{array}{c}\text { DW of reproductive } \\
\text { parts (g) }\end{array}$} & \multicolumn{3}{|c|}{ Total DW (g) } & \multirow{2}{*}{$\begin{array}{l}\text { Seed } \\
\text { yield } \\
\text { /plant } \\
\text { (g) }\end{array}$} \\
\hline & $\begin{array}{c}35 \\
\text { DAS }\end{array}$ & $\begin{array}{c}50 \\
\text { DAS }\end{array}$ & $\begin{array}{c}65 \\
\text { DAS }\end{array}$ & $\begin{array}{c}35 \\
\text { DAS }\end{array}$ & $\begin{array}{c}\mathbf{5 0} \\
\text { DAS }\end{array}$ & $\begin{array}{c}65 \\
\text { DAS }\end{array}$ & $\begin{array}{c}35 \\
\text { DAS }\end{array}$ & $\begin{array}{c}50 \\
\text { DAS }\end{array}$ & $\begin{array}{c}65 \\
\text { DAS }\end{array}$ & $\begin{array}{c}35 \\
\text { DAS }\end{array}$ & $\begin{array}{c}\mathbf{5 0} \\
\text { DAS }\end{array}$ & $\begin{array}{c}65 \\
\text { DAS }\end{array}$ & $\begin{array}{c}35 \\
\text { DAS }\end{array}$ & $\begin{array}{c}\mathbf{5 0} \\
\text { DAS }\end{array}$ & $\begin{array}{c}65 \\
\text { DAS }\end{array}$ & \\
\hline $15^{\text {th }} \mathrm{Nov}$ & 0.07 & 0.07 & 0.39 & 0.11 & 0.22 & 0.47 & 0.24 & 0.40 & 0.59 & - & 0.04 & 0.28 & 0.43 & 0.73 & 1.73 & 2.73 \\
\hline $6^{\text {th }}$ Dec & 0.05 & 0.34 & 0.23 & 0.08 & 0.40 & 0.90 & 0.16 & 0.58 & 0.34 & - & 0.10 & 0.41 & 0.29 & 1.42 & 1.88 & 1.86 \\
\hline $27^{\text {th }}$ Dec & 0.05 & 0.12 & 0.14 & 0.08 & 0.39 & 0.65 & 0.18 & 0.39 & 0.43 & - & 0.08 & 0.24 & 0.31 & 0.98 & 1.45 & 0.35 \\
\hline S.E. $(\mathrm{m}) \pm$ & 0.003 & 0.006 & 0.005 & 0.003 & 0.004 & 0.021 & 0.001 & 0.009 & 0.004 & - & 0.003 & 0.001 & 0.001 & 0.010 & 0.013 & 0.006 \\
\hline C.D. $(P=0.05)$ & 0.008 & 0.018 & 0.015 & 0.007 & 0.012 & 0.058 & 0.003 & 0.024 & 0.011 & - & 0.009 & 0.004 & 0.002 & 0.027 & 0.036 & 0.015 \\
\hline
\end{tabular}

Table.2 Main effects of genotypes on total drymatter content and its partitioning and seed yield in lentil

\begin{tabular}{|c|c|c|c|c|c|c|c|c|c|c|c|c|c|c|c|c|}
\hline \multirow[t]{2}{*}{ Genotypes } & \multicolumn{3}{|c|}{ Root DW (g) } & \multicolumn{3}{|c|}{ Shoot DW (g) } & \multicolumn{3}{|c|}{ Leaf DW (g) } & \multicolumn{3}{|c|}{$\begin{array}{l}\text { DW of reproductive parts } \\
(\mathrm{g})\end{array}$} & \multicolumn{3}{|c|}{ Total DW (g) } & \multirow{2}{*}{$\begin{array}{c}\text { Seed } \\
\text { yield } \\
\text { /plant } \\
\text { (g) }\end{array}$} \\
\hline & $\begin{array}{c}35 \\
\text { DAS }\end{array}$ & $\begin{array}{c}50 \\
\text { DAS }\end{array}$ & $\begin{array}{c}65 \\
\text { DAS }\end{array}$ & $\begin{array}{c}35 \\
\text { DAS }\end{array}$ & $\begin{array}{c}\text { 50 } \\
\text { DAS }\end{array}$ & $\begin{array}{c}65 \\
\text { DAS }\end{array}$ & $\begin{array}{c}35 \\
\text { DAS }\end{array}$ & $\begin{array}{c}\mathbf{5 0} \\
\text { DAS }\end{array}$ & $\begin{array}{c}65 \\
\text { DAS }\end{array}$ & $\begin{array}{c}35 \\
\text { DAS }\end{array}$ & $\begin{array}{c}\text { 50 } \\
\text { DAS }\end{array}$ & $\begin{array}{c}65 \\
\text { DAS }\end{array}$ & $\begin{array}{c}35 \\
\text { DAS }\end{array}$ & $\begin{array}{c}\text { 50 } \\
\text { DAS }\end{array}$ & $\begin{array}{l}65 \\
\text { DAS }\end{array}$ & \\
\hline KLS-218 & 0.04 & 0.17 & 0.28 & 0.06 & 0.25 & 0.66 & 0.13 & 0.36 & 0.42 & - & 0.02 & 0.23 & 0.23 & 0.80 & 1.58 & 1.82 \\
\hline ILL-8108 & 0.09 & 0.23 & 0.34 & 0.13 & 0.51 & 1.31 & 0.31 & 0.62 & 0.69 & - & 0.13 & 0.37 & 0.53 & 1.49 & 2.72 & 1.03 \\
\hline RL-12-171 & 0.05 & 0.14 & 0.22 & 0.07 & 0.27 & 0.58 & 0.16 & 0.35 & 0.41 & - & 0.00 & 0.17 & 0.27 & 0.77 & 1.38 & 0.78 \\
\hline BM-6 & 0.05 & 0.19 & 0.25 & 0.07 & 0.26 & 0.59 & 0.15 & 0.34 & 0.37 & - & 0.15 & 0.31 & 0.27 & 0.94 & 1.51 & 1.51 \\
\hline HULL-57 & 0.07 & 0.14 & 0.24 & 0.07 & 0.27 & 0.49 & 0.17 & 0.36 & 0.47 & - & 0.01 & 0.07 & 0.30 & 0.78 & 1.26 & 1.92 \\
\hline L-13-113 & 0.06 & 0.15 & 0.25 & 0.16 & 0.47 & 0.66 & 0.27 & 0.60 & 0.49 & - & 0.24 & 0.53 & 0.49 & 1.46 & 1.93 & 2.17 \\
\hline ILL-6002 & 0.09 & 0.27 & 0.30 & 0.13 & 0.50 & 0.99 & 0.27 & 0.68 & 0.68 & - & 0.02 & 0.43 & 0.49 & 1.46 & 2.40 & 1.74 \\
\hline ILL-10893 & 0.10 & 0.26 & 0.29 & 0.12 & 0.49 & 0.78 & 0.26 & 0.64 & 0.53 & - & 0.04 & 0.27 & 0.48 & 1.42 & 1.87 & 2.05 \\
\hline PRECOZ & 0.09 & 0.23 & 0.31 & 0.11 & 0.40 & 1.02 & 0.27 & 0.55 & 0.62 & - & 0.01 & 0.28 & 0.47 & 1.18 & 2.23 & 2.47 \\
\hline RL-12-178 & 0.06 & 0.16 & 0.28 & 0.09 & 0.31 & 0.69 & 0.19 & 0.42 & 0.48 & - & 0.06 & 0.29 & 0.33 & 0.94 & 1.74 & 1.65 \\
\hline L-4076 & 0.05 & 0.16 & 0.22 & 0.08 & 0.34 & 0.79 & 0.19 & 0.48 & 0.56 & - & 0.01 & 0.20 & 0.32 & 0.99 & 1.76 & 1.22 \\
\hline WBL-77 & 0.05 & 0.17 & 0.20 & 0.08 & 0.36 & 0.42 & 0.18 & 0.49 & 0.44 & - & 0.08 & 0.28 & 0.31 & 1.10 & 1.34 & 1.94 \\
\hline L-13-123 & 0.04 & 0.16 & 0.21 & 0.08 & 0.27 & 0.46 & 0.14 & 0.36 & 0.36 & - & 0.16 & 0.37 & 0.26 & 0.95 & 1.41 & 2.30 \\
\hline RANJAN & 0.07 & 0.13 & 0.24 & 0.07 & 0.32 & 0.70 & 0.18 & 0.47 & 0.44 & - & 0.16 & 0.43 & 0.32 & 1.08 & 1.81 & 1.28 \\
\hline Subrata & 0.04 & 0.15 & 0.25 & 0.07 & 0.29 & 0.63 & 0.16 & 0.38 & 0.42 & - & 0.04 & 0.34 & 0.27 & 0.85 & 1.63 & 1.06 \\
\hline ILL-10951 & 0.05 & 0.19 & 0.23 & 0.06 & 0.27 & 0.47 & 0.14 & 0.38 & 0.33 & - & 0.06 & 0.20 & 0.26 & 0.90 & 1.23 & 0.91 \\
\hline WBL-58 & 0.05 & 0.16 & 0.24 & 0.06 & 0.30 & 0.39 & 0.15 & 0.43 & 0.30 & - & 0.02 & 0.25 & 0.26 & 0.92 & 1.19 & 2.99 \\
\hline Asha & 0.05 & 0.14 & 0.24 & 0.13 & 0.36 & 0.57 & 0.19 & 0.47 & 0.38 & - & 0.14 & 0.50 & 0.37 & 1.12 & 1.69 & 0.81 \\
\hline BM-7 & 0.05 & 0.14 & 0.21 & 0.09 & 0.24 & 0.59 & 0.18 & 0.32 & 0.31 & - & 0.12 & 0.46 & 0.31 & 0.81 & 1.56 & 1.92 \\
\hline DPL-62 & 0.05 & 0.18 & 0.23 & 0.07 & 0.25 & 0.72 & 0.16 & 0.45 & 0.40 & - & 0.04 & 0.14 & 0.29 & 0.92 & 1.50 & 1.36 \\
\hline S.E. $(m) \pm$ & 0.004 & 0.011 & 0.016 & 0.006 & 0.019 & 0.043 & 0.012 & 0.028 & 0.026 & - & 0.012 & 0.023 & 0.019 & 0.039 & 0.063 & 0.015 \\
\hline C.D.(P=0.05) & 0.008 & 0.022 & 0.032 & 0.012 & 0.038 & 0.085 & 0.024 & 0.056 & 0.052 & - & 0.023 & 0.049 & 0.037 & 0.077 & 0.125 & 0.030 \\
\hline
\end{tabular}


Table.3 Interaction of sowing dates and genotypes on total drymatter content and its partitioning and seed yield in lentil

\begin{tabular}{|c|c|c|c|c|c|c|c|c|c|c|c|c|c|c|c|c|}
\hline \multirow[t]{2}{*}{ Interactions } & \multicolumn{3}{|c|}{ Root DW (g) } & \multicolumn{3}{|c|}{ Shoot DW (g) } & \multicolumn{3}{|c|}{ Leaf DW (g) } & \multicolumn{3}{|c|}{$\begin{array}{c}\text { DW of reproductive } \\
\text { parts }(\mathrm{g})\end{array}$} & \multicolumn{3}{|c|}{ Total DW (g) } & \multirow[t]{2}{*}{$\begin{array}{l}\text { Seed yield } \\
\text { /plant (g) }\end{array}$} \\
\hline & $\begin{array}{c}35 \\
\text { DAS }\end{array}$ & $\begin{array}{c}50 \\
\text { DAS }\end{array}$ & $\begin{array}{c}65 \\
\text { DAS }\end{array}$ & $\begin{array}{c}35 \\
\text { DAS }\end{array}$ & $\begin{array}{c}50 \\
\text { DAS }\end{array}$ & $\begin{array}{c}65 \\
\text { DAS }\end{array}$ & $\begin{array}{c}35 \\
\text { DAS }\end{array}$ & $\begin{array}{c}50 \\
\text { DAS }\end{array}$ & $\begin{array}{c}65 \\
\text { DAS }\end{array}$ & $\begin{array}{c}35 \\
\text { DAS }\end{array}$ & $\begin{array}{c}50 \\
\text { DAS }\end{array}$ & 65 DAS & $\begin{array}{c}35 \\
\text { DAS }\end{array}$ & $\begin{array}{c}\mathbf{5 0} \\
\text { DAS }\end{array}$ & $\begin{array}{c}65 \\
\text { DAS }\end{array}$ & \\
\hline S1 x g1 & 0.06 & 0.07 & 0.42 & 0.06 & 0.17 & 0.47 & 0.12 & 0.33 & 0.58 & - & 0.00 & 0.28 & 0.24 & 0.57 & 1.75 & 3.16 \\
\hline S1 x g2 & 0.10 & 0.07 & 0.39 & 0.18 & 0.35 & 0.67 & 0.45 & 0.52 & 0.57 & - & 0.19 & 0.29 & 0.73 & 1.14 & 1.91 & 1.61 \\
\hline S1 x g3 & 0.04 & 0.07 & 0.37 & 0.07 & 0.12 & 0.33 & 0.16 & 0.23 & 0.79 & - & 0.00 & 0.25 & 0.27 & 0.42 & 1.74 & 1.24 \\
\hline S1 x g4 & 0.06 & 0.03 & 0.40 & 0.08 & 0.15 & 0.39 & 0.19 & 0.26 & 0.56 & - & 0.17 & 0.18 & 0.33 & 0.61 & 1.53 & 2.99 \\
\hline S1 x g5 & 0.11 & 0.05 & 0.40 & 0.07 & 0.16 & 0.25 & 0.20 & 0.31 & 0.44 & - & 0.00 & 0.06 & 0.38 & 0.52 & 1.15 & 3.35 \\
\hline S1 x g6 & 0.05 & 0.04 & 0.35 & 0.22 & 0.27 & 0.57 & 0.31 & 0.32 & 0.47 & - & 0.19 & 0.41 & 0.59 & 0.81 & 1.79 & 2.64 \\
\hline S1 x g7 & 0.13 & 0.08 & 0.37 & 0.17 & 0.26 & 0.43 & 0.35 & 0.48 & 0.52 & - & 0.00 & 0.33 & 0.65 & 0.81 & 1.65 & 2.13 \\
\hline S1 x g8 & 0.11 & 0.11 & 0.43 & 0.18 & 0.40 & 0.60 & 0.35 & 0.57 & 0.57 & - & 0.00 & 0.30 & 0.65 & 1.08 & 1.90 & 2.29 \\
\hline S1 x g9 & 0.11 & 0.14 & 0.46 & 0.14 & 0.35 & 0.97 & 0.35 & 0.52 & 0.89 & - & 0.00 & 0.31 & 0.60 & 1.02 & 2.63 & 4.77 \\
\hline S1 x g10 & 0.07 & 0.06 & 0.43 & 0.11 & 0.20 & 0.56 & 0.19 & 0.39 & 0.74 & - & 0.00 & 0.31 & 0.37 & 0.65 & 2.04 & 3.16 \\
\hline S1 x g11 & 0.05 & 0.05 & 0.36 & 0.12 & 0.22 & 0.51 & 0.25 & 0.39 & 0.57 & - & 0.00 & 0.29 & 0.42 & 0.66 & 1.73 & 2.66 \\
\hline S1 x g12 & 0.08 & 0.08 & 0.36 & 0.10 & 0.24 & 0.42 & 0.21 & 0.55 & 0.60 & - & 0.00 & 0.29 & 0.39 & 0.86 & 1.67 & 3.83 \\
\hline S1 x g13 & 0.06 & 0.05 & 0.36 & 0.11 & 0.17 & 0.47 & 0.16 & 0.30 & 0.55 & - & 0.06 & 0.30 & 0.32 & 0.57 & 1.67 & 3.99 \\
\hline S1 x g 14 & 0.07 & 0.05 & 0.36 & 0.11 & 0.24 & 0.48 & 0.26 & 0.47 & 0.61 & - & 0.14 & 0.31 & 0.43 & 0.89 & 1.76 & 2.17 \\
\hline S1 x g15 & 0.05 & 0.07 & 0.42 & 0.07 & 0.17 & 0.57 & 0.19 & 0.34 & 0.75 & - & 0.00 & 0.33 & 0.32 & 0.58 & 2.07 & 1.93 \\
\hline S1 x g16 & 0.07 & 0.11 & 0.37 & 0.06 & 0.14 & 0.23 & 0.16 & 0.30 & 0.42 & - & 0.00 & 0.31 & 0.30 & 0.55 & 1.33 & 1.43 \\
\hline S1 x g17 & 0.06 & 0.08 & 0.42 & 0.08 & 0.18 & 0.23 & 0.20 & 0.39 & 0.48 & - & 0.00 & 0.28 & 0.34 & 0.64 & 1.41 & 4.99 \\
\hline S1 x g18 & 0.07 & 0.06 & 0.35 & 0.10 & 0.27 & 0.57 & 0.26 & 0.54 & 0.73 & - & 0.03 & 0.30 & 0.43 & 0.90 & 1.95 & 1.24 \\
\hline S1 x g19 & 0.06 & 0.07 & 0.38 & 0.13 & 0.13 & 0.35 & 0.29 & 0.29 & 0.51 & - & 0.05 & 0.29 & 0.48 & 0.54 & 1.52 & 3.07 \\
\hline S1 x g20 & 0.05 & 0.07 & 0.38 & 0.09 & 0.21 & 0.38 & 0.17 & 0.41 & 0.50 & - & 0.00 & 0.10 & 0.32 & 0.69 & 1.36 & 2.01 \\
\hline S.E.(m) \pm & 0.008 & 0.019 & 0.028 & 0.011 & 0.032 & 0.075 & 0.021 & 0.048 & 0.045 & - & 0.020 & 0.042 & 0.032 & 0.066 & 0.107 & 0.026 \\
\hline C.D. $(P=0.05)$ & 0.016 & 0.040 & 0.056 & 0.022 & 0.064 & 0.154 & 0.041 & 0.097 & 0.089 & - & 0.040 & 0.083 & 0.063 & 0.132 & 0.214 & 0.053 \\
\hline
\end{tabular}




\begin{tabular}{|c|c|c|c|c|c|c|c|c|c|c|c|c|c|c|c|c|}
\hline \multirow[t]{2}{*}{ Interactions } & \multicolumn{3}{|c|}{ Root DW (g) } & \multicolumn{3}{|c|}{ Shoot DW (g) } & \multicolumn{3}{|c|}{ Leaf DW (g) } & \multicolumn{3}{|c|}{$\begin{array}{c}\text { DW of reproductive } \\
\text { parts }(\mathrm{g})\end{array}$} & \multicolumn{3}{|c|}{ Total DW (g) } & \multirow{2}{*}{$\begin{array}{c}\text { Seed } \\
\text { yield } \\
\text { /plant (g) }\end{array}$} \\
\hline & $\begin{array}{c}35 \\
\text { DAS }\end{array}$ & $\begin{array}{c}\mathbf{5 0} \\
\text { DAS }\end{array}$ & $\begin{array}{c}65 \\
\text { DAS }\end{array}$ & $\begin{array}{c}35 \\
\text { DAS }\end{array}$ & $\begin{array}{c}\mathbf{5 0} \\
\text { DAS }\end{array}$ & $\begin{array}{c}65 \\
\text { DAS }\end{array}$ & $\begin{array}{c}35 \\
\text { DAS }\end{array}$ & $\begin{array}{c}\mathbf{5 0} \\
\text { DAS }\end{array}$ & $\begin{array}{c}65 \\
\text { DAS }\end{array}$ & $\begin{array}{c}35 \\
\text { DAS }\end{array}$ & $\begin{array}{c}50 \\
\text { DAS }\end{array}$ & $\begin{array}{c}65 \\
\text { DAS }\end{array}$ & $\begin{array}{c}35 \\
\text { DAS }\end{array}$ & $\begin{array}{c}\mathbf{5 0} \\
\text { DAS }\end{array}$ & $\begin{array}{c}65 \\
\text { DAS }\end{array}$ & \\
\hline S2 $\times$ g1 & 0.04 & 0.35 & 0.24 & 0.05 & 0.33 & 0.86 & 0.11 & 0.50 & 0.30 & - & 0.00 & 0.12 & 0.20 & 1.18 & 1.52 & 2.21 \\
\hline S2 2 g2 & 0.09 & 0.48 & 0.41 & 0.15 & 0.65 & 1.93 & 0.33 & 0.80 & 0.32 & - & 0.17 & 0.60 & 0.56 & 2.09 & 3.27 & 1.15 \\
\hline S2 $x$ g3 & 0.05 & 0.29 & 0.19 & 0.04 & 0.36 & 0.90 & 0.11 & 0.55 & 0.33 & - & 0.00 & 0.14 & 0.21 & 1.21 & 1.55 & 0.09 \\
\hline S2 $\times \mathrm{g} 4$ & 0.03 & 0.45 & 0.21 & 0.05 & 0.39 & 0.92 & 0.09 & 0.58 & 0.35 & - & 0.11 & 0.50 & 0.17 & 1.52 & 1.99 & 1.10 \\
\hline S2 $\times$ g5 & 0.06 & 0.27 & 0.18 & 0.04 & 0.34 & 0.49 & 0.11 & 0.51 & 0.46 & - & 0.00 & 0.04 & 0.20 & 1.13 & 1.17 & 2.35 \\
\hline S2 x g6 & 0.05 & 0.28 & 0.27 & 0.16 & 0.53 & 0.79 & 0.26 & 0.84 & 0.48 & - & 0.21 & 0.87 & 0.47 & 1.86 & 2.42 & 2.83 \\
\hline S2 x g7 & 0.05 & 0.50 & 0.34 & 0.10 & 0.55 & 1.51 & 0.21 & 0.82 & 0.47 & - & 0.00 & 0.67 & 0.35 & 1.86 & 2.99 & 2.86 \\
\hline S2 x g8 & 0.12 & 0.46 & 0.29 & 0.11 & 0.53 & 0.78 & 0.24 & 0.69 & 0.46 & - & 0.11 & 0.30 & 0.47 & 1.79 & 1.83 & 3.05 \\
\hline S2 $x$ g9 & 0.06 & 0.42 & 0.31 & 0.09 & 0.49 & 1.58 & 0.23 & 0.76 & 0.47 & - & 0.00 & 0.49 & 0.38 & 1.67 & 2.85 & 1.83 \\
\hline S2 $\times$ g10 & 0.05 & 0.29 & 0.29 & 0.07 & 0.41 & 0.82 & 0.16 & 0.60 & 0.28 & - & 0.16 & 0.37 & 0.29 & 1.47 & 1.76 & 1.56 \\
\hline S2 x g11 & 0.05 & 0.31 & 0.13 & 0.07 & 0.36 & 0.80 & 0.15 & 0.54 & 0.45 & - & 0.02 & 0.17 & 0.27 & 1.23 & 1.54 & 0.94 \\
\hline S2 x g12 & 0.04 & 0.28 & 0.13 & 0.08 & 0.34 & 0.35 & 0.18 & 0.43 & 0.43 & - & 0.15 & 0.36 & 0.29 & 1.21 & 1.27 & 1.56 \\
\hline S2 x g13 & 0.03 & 0.34 & 0.16 & 0.04 & 0.35 & 0.53 & 0.08 & 0.47 & 0.37 & - & 0.21 & 0.58 & 0.15 & 1.36 & 1.63 & 2.13 \\
\hline S2 x g 14 & 0.07 & 0.27 & 0.22 & 0.06 & 0.38 & 0.74 & 0.17 & 0.57 & 0.34 & - & 0.21 & 0.48 & 0.30 & 1.43 & 1.78 & 1.31 \\
\hline S2 x g15 & 0.03 & 0.26 & 0.20 & 0.06 & 0.30 & 0.85 & 0.14 & 0.44 & 0.18 & - & 0.00 & 0.19 & 0.23 & 0.99 & 1.42 & 1.05 \\
\hline S2 x g16 & 0.05 & 0.38 & 0.21 & 0.06 & 0.31 & 0.81 & 0.16 & 0.47 & 0.29 & - & 0.17 & 0.18 & 0.27 & 1.33 & 1.49 & 1.20 \\
\hline S2 $\times$ g17 & 0.03 & 0.30 & 0.18 & 0.04 & 0.29 & 0.51 & 0.09 & 0.44 & 0.17 & - & 0.02 & 0.19 & 0.16 & 1.05 & 1.06 & 3.84 \\
\hline S2 x g18 & 0.04 & 0.29 & 0.27 & 0.21 & 0.35 & 0.67 & 0.14 & 0.52 & 0.20 & - & 0.23 & 1.00 & 0.39 & 1.39 & 2.14 & 1.09 \\
\hline S2x g19 & 0.04 & 0.26 & 0.15 & 0.05 & 0.37 & 1.20 & 0.10 & 0.50 & 0.27 & - & 0.19 & 0.76 & 0.19 & 1.31 & 2.38 & 2.58 \\
\hline S2 x g20 & 0.05 & 0.36 & 0.19 & 0.06 & 0.40 & 1.03 & 0.15 & 0.64 & 0.24 & - & 0.01 & 0.10 & 0.26 & 1.41 & 1.56 & 1.67 \\
\hline S.E. $(\mathrm{m}) \pm$ & 0.008 & 0.019 & 0.028 & 0.011 & 0.032 & 0.075 & 0.021 & 0.048 & 0.045 & - & 0.020 & 0.042 & 0.032 & 0.066 & 0.107 & 0.026 \\
\hline C.D. $(P=0.05)$ & 0.016 & 0.040 & 0.056 & 0.022 & 0.064 & 0.154 & 0.041 & 0.097 & 0.089 & - & 0.040 & 0.083 & 0.063 & 0.132 & 0.214 & 0.053 \\
\hline
\end{tabular}




\begin{tabular}{|c|c|c|c|c|c|c|c|c|c|c|c|c|c|c|c|c|}
\hline \multirow[t]{2}{*}{ Interactions } & \multicolumn{3}{|c|}{ Root DW (g) } & \multicolumn{3}{|c|}{ Shoot DW (g) } & \multicolumn{3}{|c|}{ Leaf DW (g) } & \multicolumn{3}{|c|}{$\begin{array}{c}\text { DW of reproductive } \\
\text { parts (g) }\end{array}$} & \multicolumn{3}{|c|}{ Total DW (g) } & \multirow{2}{*}{$\begin{array}{l}\text { Seed } \\
\text { yield } \\
\text { /plant } \\
(\mathrm{g})\end{array}$} \\
\hline & $\begin{array}{c}35 \\
\text { DAS }\end{array}$ & $\begin{array}{c}50 \\
\text { DAS }\end{array}$ & $\begin{array}{c}65 \\
\text { DAS }\end{array}$ & $\begin{array}{c}35 \\
\text { DAS }\end{array}$ & $\begin{array}{c}50 \\
\text { DAS }\end{array}$ & $\begin{array}{c}65 \\
\text { DAS }\end{array}$ & $\begin{array}{c}35 \\
\text { DAS }\end{array}$ & $\begin{array}{c}50 \\
\text { DAS }\end{array}$ & $\begin{array}{c}65 \\
\text { DAS }\end{array}$ & $\begin{array}{c}35 \\
\text { DAS }\end{array}$ & $\begin{array}{c}50 \\
\text { DAS }\end{array}$ & 65 DAS & $\begin{array}{c}35 \\
\text { DAS }\end{array}$ & $\begin{array}{c}50 \\
\text { DAS }\end{array}$ & $\begin{array}{c}65 \\
\text { DAS }\end{array}$ & \\
\hline S3 x g1 & 0.03 & 0.10 & 0.18 & 0.07 & 0.25 & 0.64 & 0.16 & 0.25 & 0.38 & - & 0.05 & 0.28 & 0.23 & 0.64 & 1.47 & 0.09 \\
\hline S3 x g2 & 0.07 & 0.15 & 0.23 & 0.07 & 0.53 & 1.34 & 0.16 & 0.53 & 1.19 & - & 0.03 & 0.23 & 0.30 & 1.25 & 2.98 & 0.33 \\
\hline S3 x g3 & 0.05 & 0.07 & 0.12 & 0.09 & 0.33 & 0.51 & 0.21 & 0.27 & 0.12 & - & 0.01 & 0.12 & 0.35 & 0.69 & 0.86 & 0.20 \\
\hline S3 x g4 & 0.06 & 0.09 & 0.13 & 0.07 & 0.24 & 0.45 & 0.18 & 0.18 & 0.19 & - & 0.16 & 0.24 & 0.31 & 0.67 & 1.01 & 0.45 \\
\hline S3 x g5 & 0.03 & 0.10 & 0.13 & 0.09 & 0.32 & 0.72 & 0.19 & 0.26 & 0.51 & - & 0.02 & 0.12 & 0.32 & 0.69 & 1.48 & 0.05 \\
\hline S3 x g6 & 0.08 & 0.13 & 0.13 & 0.10 & 0.62 & 0.60 & 0.23 & 0.64 & 0.51 & - & 0.31 & 0.32 & 0.41 & 1.70 & 1.57 & 1.05 \\
\hline S3 x g7 & 0.09 & 0.24 & 0.20 & 0.12 & 0.69 & 1.03 & 0.27 & 0.74 & 1.04 & - & 0.05 & 0.29 & 0.47 & 1.72 & 2.56 & 0.23 \\
\hline S3 x g8 & 0.05 & 0.21 & 0.15 & 0.08 & 0.52 & 0.96 & 0.20 & 0.66 & 0.56 & - & 0.02 & 0.21 & 0.33 & 1.41 & 1.89 & 0.80 \\
\hline S3 x g9 & 0.10 & 0.14 & 0.15 & 0.10 & 0.35 & 0.52 & 0.24 & 0.36 & 0.49 & - & 0.02 & 0.04 & 0.44 & 0.87 & 1.20 & 0.81 \\
\hline S3 x g10 & 0.06 & 0.12 & 0.12 & 0.08 & 0.31 & 0.71 & 0.21 & 0.24 & 0.41 & - & 0.02 & 0.18 & 0.35 & 0.70 & 1.42 & 0.23 \\
\hline S3 x g11 & 0.05 & 0.14 & 0.16 & 0.06 & 0.43 & 1.05 & 0.16 & 0.51 & 0.68 & - & 0.01 & 0.14 & 0.26 & 1.09 & 2.02 & 0.05 \\
\hline S3 x g12 & 0.03 & 0.15 & 0.12 & 0.06 & 0.50 & 0.49 & 0.15 & 0.48 & 0.28 & - & 0.09 & 0.18 & 0.24 & 1.22 & 1.07 & 0.44 \\
\hline S3 x g13 & 0.05 & 0.11 & 0.12 & 0.09 & 0.30 & 0.38 & 0.18 & 0.29 & 0.17 & - & 0.21 & 0.25 & 0.32 & 0.90 & 0.92 & 0.79 \\
\hline S3 x g 14 & 0.05 & 0.08 & 0.14 & 0.05 & 0.35 & 0.88 & 0.13 & 0.37 & 0.38 & - & 0.12 & 0.50 & 0.24 & 0.93 & 1.90 & 0.35 \\
\hline S3 x g15 & 0.04 & 0.12 & 0.13 & 0.07 & 0.38 & 0.46 & 0.14 & 0.36 & 0.33 & - & 0.12 & 0.49 & 0.25 & 0.98 & 1.41 & 0.20 \\
\hline S3 x g16 & 0.03 & 0.09 & 0.11 & 0.06 & 0.35 & 0.36 & 0.11 & 0.38 & 0.29 & - & 0.02 & 0.12 & 0.20 & 0.84 & 0.88 & 0.13 \\
\hline S3 x g17 & 0.05 & 0.11 & 0.11 & 0.06 & 0.44 & 0.44 & 0.17 & 0.47 & 0.26 & - & 0.05 & 0.27 & 0.28 & 1.07 & 1.08 & 0.13 \\
\hline S3 x g18 & 0.04 & 0.08 & 0.11 & 0.07 & 0.46 & 0.46 & 0.17 & 0.35 & 0.20 & - & 0.17 & 0.20 & 0.28 & 1.07 & 0.96 & 0.11 \\
\hline S3 x g19 & 0.05 & 0.09 & 0.10 & 0.07 & 0.23 & 0.23 & 0.15 & 0.17 & 0.13 & - & 0.11 & 0.32 & 0.27 & 0.59 & 0.78 & 0.12 \\
\hline S13 x g20 & 0.05 & 0.11 & 0.13 & 0.07 & 0.13 & 0.76 & 0.17 & 0.31 & 0.46 & - & 0.11 & 0.23 & 0.29 & 0.66 & 1.57 & 0.42 \\
\hline S.E.(m) \pm & 0.008 & 0.019 & 0.028 & 0.011 & 0.032 & 0.075 & 0.021 & 0.048 & 0.045 & - & 0.020 & 0.042 & 0.032 & 0.066 & 0.107 & 0.026 \\
\hline $\begin{array}{l}\text { C.D. } \\
(P=0.05)\end{array}$ & 0.016 & 0.040 & 0.056 & 0.022 & 0.064 & 0.154 & 0.041 & 0.097 & 0.089 & - & 0.040 & 0.083 & 0.063 & 0.132 & 0.214 & 0.053 \\
\hline
\end{tabular}


In case of shoot dry weight, there was a steep rise between 35 and 50 DAS in all the genotypes. Out of all the genotypes, ILL-8108 registered the highest mean for total dry weight at 65 DAS along with drymatter content in root, shoot and leaf at 65 DAS. The highest accumulation of drymatter in reproductive sink at 65 DAS was registered by L-13-113. It might indicate the earliness in flowering of this genotype under all sowing dates among all the genotypes. The genotypes WBL-58, ILL-10951 and HULL-57 exhibited the lowest mean for total drymatter content in plant at 65 DAS. HULL-57 also showed the minimum accumulation of drymatter in reproductive sink at 65 DAS among all the twenty genotypes.

The data on interaction of sowing dates and genotypes for different growth parameters have been presented in Table 3. Perusal of the data indicated that all but six genotypes registered the significant decrease in plant dry weight at 65 DAS under $3^{\text {rd }}$ sowing date as compared to $1^{\text {st }}$ sowing. Out of all the genotypes, ILL-8108 showed maximum increase $\left(56.02 \%\right.$ over $1^{\text {st }}$ sowing date) in dry weight under the latest sowing date and it was closely followed by ILL-6002.

On the contrary, PRECOZ recorded the highest decrease $(54.37 \%)$ in plant dry weight at 65 DAS under $3^{\text {rd }}$ sowing date and it was closely followed by RL-12-171 and Asha. Thus, summarizing the data it might be concluded that sowing of the genotypes on $27^{\text {th }}$ December led to overall reduction in the drymatter content in the plant as well the drymatter partitioned to the reproductive sinks at 65 DAS. There was genotypic variation in regard to the extent of reduction. The results corroborated the early finding of Haq et al., (2012) who also reported decline in biomass production in lentil under osmotic stress in late sowing condition. In the experiment, the twenty genotypes of lentil registered gradual decline in seed yield concomitantly with the late sowing dates and showed the most drastic reduction under $3^{\text {rd }}$ sowing date. This was caused by reduced soil moisture and high air temperature, especially, during the reproductive stage of the crop. Significant reduction in seed yield under water deficit was reported earlier by Shrestha et al., (2006), Azizi et al., (2009) and Panahyan et al., (2009). Delahunty et al., (2015) also reported yield reduction in lentil as a result of heat stress. The seed yield/plant varied from $0.78 \mathrm{~g}$ to $2.47 \mathrm{~g}$ averaged over sowing dates. The two genotypes, PRECOZ and L-13-123 with mean seed yield of $2.47 \mathrm{~g} / \mathrm{plant}$ and 2.30 $\mathrm{g} /$ plant, respectively, registered the highest mean value while RL-12-171 had the lowest seed yield/plant.

Out of all the genotypes, KLS-218, HULL-57, and L-4076 with 97.15, 98.51 and $98.12 \%$ reduction in seed yield/plant under $3^{\text {rd }}$ sowing date, respectively, were found to be the most sensitive to terminal drought and heat stress in the present experiment.

Roots and shoots together constitute the entire plant structure. The optimum proportion of dry mass between these parts should be partitioned if final yield to be maximized (Gorney and Larson, 1989). Several researchers reported that the genotypes which produced greater total drymatter showed higher yield due to large root system and leaf area (Goutam and Sharma, 1987; Flood et al., 1995; Mondal et al., 2011). Mondal et al., (2014) concluded that percent contribution of dry mass partitioning into different plant parts had no significant influence on seed yield but leaf and stem dry mass production had influence on seed yield in lentil.

\section{Acknowledgement}

Authors are grateful to ICARDA for supporting this research. 


\section{References}

Azizi-e-Chakherchaman, S., Mostafaei, H., Yari, A., Hassanzadeh, M., Jamaati-eSomarin, S. and Easazadeh, R. 2009. Study of relationships of leaf Relative Water Content, Cell Membrane Stability and duration of growth period with grain yield of lentil under rain-fed and irrigated conditions. Research Journal of Biological Sciences. 4: 842847.

Delahunty, A., Nuttall, J., Nicolas, M. and Brand, J. 2015. Genotypic heat tolerance in lentil. 17 ASA Conference. 20-24 September. Hobart. Australia.

Flood, R.G., Martin, P.J. and Gardner, W.K. 1995. Drymatter accumulation and partitioning and its relationship to grain yield in wheat. Australian Journal of Experimental Agriculture. 35: 495-502.

Gautom, R.C. and Sharma, K.C. 1987. Drymatter accumulation under different planting schemes and plant densities of rice. Indian Journal of Agricultural Research. 21: 101-109.

Gorney, A.G. and Larson, S. 1989. New aspect in root breeding. Proceeding III Congress of Eucarpia, Goltingen, Germany. Pp. 339-356.

Haq, A. Vamil, R. and Agnihotri, R.K. 2012. Effect of moisture stress (PEG) on biomass, total chlorophyll and proline content of lentil (Lens culinaris
Medik.). Agricultural-and-BiologicalResearch. 28: 56-63.

Mondal, M.M.A., Fakir, M.S.A., Islam, M.N. and Samad, M.A. 2011. Physiology of seed yield in mungbean: growth and drymatter production. Bangladesh Journal of Botany.40: 133-138.

Mondal, M.M.A., Putch, A.B., Kashem, M.A., Haque, M.A. and Razzaque, A.H.M. 2014. Growth and dry mass partitioning in plant parts of lentil. Legume Research. 37(4): 434-438.

Mondal, M.M.A., Putch, A.B., Malek, M.A., Roy, S. and Rafii, M.Y. 2013. Contribution of morpho-physiological traits on yield of lentil. Australian Journal of Crop Science. 7: 1167-1172.

Panahyan-e-Kivi, M., Ebadi, A., Tobeh, A. and Jamaati-e-Somarin, S. 2009. Evaluation of yield and yield components of lentil genotypes under drought stress. Research-Journal-ofEnvironmental-Sciences. 3: 456-460.

SAIC (South Asian Agriculture Information Centre). 2010. Annual report of 200910. SAARC centre, farm gate, Dhaka1215. Bangladesh. Pp.117.

Shrestha, R., Turner, N.C., Siddique, K.H.M., Turner, D.W. and Speijers, J. 2006. A water deficit during pod development $\mathrm{n}$ lentils reduce flower and pod numbers but not seed size. Australian Journal of Agricultural Research. 57: 427-438.

\section{How to cite this article:}

Ananya Baidya, Anjan Kumar Pal, Mohammed Anwar Ali and Rajib Nath. 2018. Impact of Sowing Dates on Drymatter Production, Partitioning and Yield in Lentil (Lens culinaris Medikus). Int.J.Curr.Microbiol.App.Sci. 7(04): 3122-3129.

doi: https://doi.org/10.20546/ijcmas.2018.704.354 